\title{
3 Research Square

\section{The Vent-Lock Protocol: 3D Printing and Testing of A Ventilator Multiplexer to Enhance the Capacity of Treating Patients with COVID-19}

Helen Xun ( $\sim$ hxun1@jhmi.edu )

Department of Plastic and Reconstructive Surgery, Johns Hopkins School of Medicine https://orcid.org/0000-0002-0412-4356

Christopher Shallal

Department of Biomedical Engineering

Justin Unger

Department of Civil and Systems Engineering, Johns Hopkins University

Runhan Tao

Department of Biomedical Engineering, Johns Hopkins University

Alberto Torres

Department of Civil and Systems Engineering, Johns Hopkins University, Baltimore

Michael Vladimirov

Department of Civil and Systems Engineering, Johns Hopkins University

Jenna Frye

Maryland Institute College of Arts

Mohit Singhala

Department of Mechanical Engineering, Johns Hopkins University

Brockett Horne

Maryland Institute College of Arts

Pooja Yesantharao

Department of Plastic and Reconstructive Surgery, Johns Hopkins School of Medicine

Bo Soo Kim

Johns Hopkins School of Medicine

Broc Burke

Washington University in St. Louis School of Medicine

\section{Michael Montana}

Washington University in St. Louis School of Medicine

Michael Talcott

Washington University in St. Louis School of Medicine

\section{Bradford Winters}

Johns Hopkins School of Medicine 


\section{Margaret Frisella}

Washington University in St. Louis School of Medicine

\section{Bradley Kushner}

Washington University in St. Louis School of Medicine

\section{Justin Sacks}

Washington University in St. Louis School of Medicine

\section{James Guest}

Department of Civil and Systems Engineering, Johns Hopkins University

\section{Sung Hoon Kang}

Department of Mechanical Engineering, Johns Hopkins University

\section{Julie Caffrey}

Johns Hopkins School of Medicine

\section{Method Article}

Keywords: ventilators, mechanical ventilators, anesthesia gas machines, anesthesia gas, acute respiratory distress syndrome (ARDS), ventilator splitter, ventilator multiplexer, 3D printing, 3DP, flow restrictor, COVID-19, COVID19, coronavirus, SARS-CoV-2, flow restriction, airflow restriction, ventilator circuit, ventilator circuit modification, ventilator monitoring, ventilator pressure control, ventilator volume control, manometer, positive end expiratory pressure, PEEP

Posted Date: October 13th, 2020

DOI: https://doi.org/10.21203/rs.3.pex-1156/v1

License: (a) (1) This work is licensed under a Creative Commons Attribution 4.0 International License. Read Full License 


\section{Abstract}

Mechanical ventilators are essential to patients who become critically ill from acute respiratory distress syndrome (ARDS), and shortages have been reported due to the novel severe acute respiratory syndrome coronavirus 2 (SARS-CoV-2). We utilized cost-effective, on-demand 3D printing (3DP) technology to produce critical components for a novel ventilator multiplexer system, Vent-Lock, to split one ventilator or anesthesia gas machine between two patients. FloRest, a novel 3DP flow restrictor, provides clinicians control of tidal volumes and positive end expiratory pressure (PEEP), using the 3DP manometer adaptor to monitor pressures. We tested the ventilator splitter circuit in simulation centers between artificial lungs and used an anesthesia gas machine to successfully ventilate two swines. As one of the first studies to demonstrate splitting one anesthesia gas machine between two swines, we present proof-of-concept of a de novo, closed, multiplexing system, with flow restriction for individualized patient therapy. Our studies underscore that while possible, ventilator multiplexing is a complicated synergy between machine settings, circuit modification, and patient monitoring. Consequently, ventilator multiplexing is reserved only as a last emergency resource, by trained clinicians and respiratory therapists with ventilator operative experience.

\section{Introduction}

The novel severe acute respiratory syndrome coronavirus 2 (SARS-CoV-2) has led to a global pandemic resulting in rapid depletion of resources necessary to care for critically ill patients, such as mechanical ventilators and their associated parts. Mechanical ventilators are critical for the treatment of approximately $5-10 \%$ of patients with coronavirus disease (COVID-19) who become critically ill from acute respiratory distress syndrome (ARDS) ${ }^{1}$. In the face of the COVID-19 pandemic, it is estimated that there is a global ventilator shortage of $880,000^{2}$. This shortage may disproportionately affect developing countries who suffer from lack of medical infrastructure and resources ${ }^{3}$, historically resulting in higher mortality rates in pandemics such as the Spanish Flu ${ }^{4}$. For example, the continent of Africa has limited ventilator capacity, with only 2,000 ventilators across 41 countries $^{5}$. This capacity deficit is further worsened by the increased need due to the COVID-19 pandemic.

Ventilator shortages occur in resource-rich countries as well. Previous disasters and current projections suggest that hospitals may be operating at $120-160 \%$ capacity in the face of a pandemic or national disaster ${ }^{6}$. Projections suggest that if $20 \%$ of the United States population is infected with the virus, there will be significant deficiencies in intensive care unit beds and mechanical ventilators ${ }^{6,7}$. Furthermore, given the potential for a second wave of infection, epidemiologists predict that if countries continue to lift restrictions used to slow the spread of the virus too early, then a second global peak may result in a further shortage of medical supplies ${ }^{8}$, ventilators and ventilator associated parts. The Society of Critical Care Medicine shared that clinicians continue to report ventilator shortages in summer of 2020, including $53 \%$ of 587 surveyed ICU clinicians did not have enough ventilators and had to use non-standard ventilators or non-invasive devices, and a small percentage declined care due to shortage of ventilators, 
or placed two patients on one ventilator ${ }^{9}$. Consequently, there is a critical need to address urgent ventilator shortages in the face of the COVID-19 pandemic.

While the introduction of more ventilators, either de novo or commercial, could solve the urgent medical needs arising from the COVID-19 pandemic, this solution represents a theoretical ideal that cannot be achieved given the current monetary, time, hospital infrastructure, limited scaling and production capacity, and supply chain constraints ${ }^{10,11}$, further exacerbated by lack of standardization of parts across brands 12. An alternative strategy to quickly increase ventilator capacity as an immediate step in urgent settings is to "split" or multiplex ventilators and anesthesia gas machines. Ventilator multiplexing allows the usage of one machine to ventilate multiple patients and effectively increases the clinical capacity to support urgent needs.

The concept of using one ventilator to support multiple patients during a disaster surge was first published in 2006 by Neyman et al. ${ }^{13}$, who reported that four patients could be supported for 12 hours using ventilator equipment and tubing. However, these in vitro studies were restricted to patients with similar body habitus and lung compliances. Despite these study limitations, it introduced ventilator multiplexing as a potential solution for emergency situations. In 2017, this proof-of-concept was demonstrated in actual patients during the 2017 Las Vegas shooting ${ }^{14}$ when a physician supported multiple patients on a single ventilator as a temporary emergency situation until more resources became available. The ability to multiplex ventilators is valuable for global preparedness mechanisms to promptly increase ventilator capacity as an immediate response for disasters, such as trauma surges, natural disasters, or in military frontlines.

Emergency use of ventilator multiplexing is dependent on the dynamic lung states of the patients, including associated lung compliances and airway resistances that drive airflow balance. In the evolving pathologic state of COVID-19 patients with ARDS, an interdependent ventilation system poses many safety concerns. The Society of Critical Care Medicine and other societies in respiratory care issued a joint statement ${ }^{15}$ summarizing main concerns with ventilator multiplexing ${ }^{16}$, including the inability to independently monitor and control ventilation parameters (volumes, pressures, rates) critical for ARDS treatment, thus risking adverse outcomes such as underventilation, or ventilator induced lung injury (VILI) such as barotrauma. Additional concerns include ventilator alarm management, disrupted balance of ventilation if a patient has spontaneous breathing, sudden deterioration, kink in the tubing, and viral contamination if breathing circuits between patients are mixed, or the circuit becomes open. While these barriers exist, they are not insurmountable for emergency ventilator multiplexing. For example, recent engineering solutions have emerged using off-the-shelf medical components that mitigate concerns in ventilator multiplexing by volume or pressure control, and monitoring ${ }^{17}$. It is optimistic that these circuits present potential solutions for ventilator shortages in emergency situations; however, deployment can be limited due to unstable supply chains that make these off-the-shelf medical components difficult to acquire ${ }^{18}$, even in developed countries up to six months from inciting events ${ }^{19}$. Consequently, rapid production of de novo ventilator multiplexing solutions are investigated to further address these barriers. 
Among multiple modalities for de novo manufacturing to address medical equipment shortages, $3 \mathrm{D}$ printing has come to the forefront during the COVID-19 pandemic to address critical shortages ${ }^{12,20}$. 3D printing is a type of additive manufacturing that has emerged in the past decades as a cost-effective, rapid on-demand production modality with broad applications due to its ability to produce intricate and complex geometries from computer-aided designs without tooling and expensive machines ${ }^{21}$. 3D printing enables faster design and manufacturing processes ${ }^{22}$, so that it can be utilized in emergency situations to fill gaps in the supply chain ${ }^{23}$. Specifically, in reference to COVID-19, there have been multiple shortages in ventilator associated equipment and valves, leading healthcare providers to look into different avenues of manufacturing to address the gap in the supply ${ }^{20}$. Ventilator splitter products which primarily use commercial medical equipment may have limited or unreliable supply ${ }^{17}$ in urgent situations. We address urgent medical needs arising from unstable supply chains by using 3D printing to rapidly and cost-effectively prototype and test components of our ventilator splitter circuit using biocompatible and sterilizable materials.

In this study, we present Vent-Lock, a de novo, ventilator multiplexing system that addresses major concerns with ventilator splitting, and is rapidly produced via 3D printing, thus tapping into a broad international production infrastructure largely unaffected by the pandemic. The Vent-Lock breathing circuit provides clinicians with a way to control, manage and monitor patients split on one ventilator; circuit components allow for the change in individual tidal volumes and positive end expiratory pressure (PEEP), pressure monitoring, and minimized back flow and risk of contamination. Our novel, air-tight, 3DP flow restrictor (FloRest) is designed to provide clinicians with precise control of tidal volumes. We validate the use of Vent-Lock FloRest for both ventilators and anesthesia gas machines, successfully ventilating simulated patients with mismatched lung compliances. Furthermore, we ventilated two swine safely with Vent-Lock, demonstrating the device's ability to both safely multiplex patients and to evolve anesthesia gas machines (which are more commonly available in developing countries as compared to ventilators) with increased ventilation settings. We share differences in multiplexing of anesthesia gas machines and ventilators, and the impact of ventilator control mode (volume control versus pressure control) on multiplexing, and additional challenges. The novel, de novo, 3DP Vent-Lock circuit and FloRest is well positioned to rapidly increase capacity of mechanical ventilators to provisionally meet ventilator shortages due to the COVID-19 pandemic and future pandemics and disasters.

\section{Reagents}

\section{D printing procedure reagents}

1. Surgical guide resin (Surgical Guide, Formlabs)

2. $>99.5 \%$ isopropyl alcohol (CAS Number: 67-63-0, Sigma Aldrich),

3. PETG Filament (PETG 3D Printer Filament, FilaMatrix, Virginia, USA) 


\section{Equipment}

\section{DP Production}

1. Stereolithography Production

- Formlabs Form 2, Form 3, or Form 3B SLA Printer

- Form Cure, Formlabs

- O-rings (E1000-212/AS568-212, O-Rings EPDM, FDA EPDM, Marco Rubber \& Plastics, Seabrook, New Hampshire, USA)

2. Fused Deposition Modeling

- Printer: e3d, BigBox3D Ltd, Oxfordshire, UK or Little Monster, Tevo 3D Electronic Technology Co. Ltd, Zhanjiang, China

- PETG Filament (PETG 3D Printer Filament, FilaMatrix, Virginia, USA).

\section{Sterilization}

1. Dry vacuum autoclave (Sr $24 \mathrm{C}$ Adv-Plus ${ }^{\mathrm{TM}}$, Consolidated Sterilizer Systems, Boston, Massachusetts, USA)

2. Oven (VO1824HPC, Lindberg/Blue M Vacuum Oven 127.4L, Thermo Scientific, Waltham, MA, USA).

3. Particle counter (SOLAIR 3100, Lighthouse Worldwide Solutions)

\section{Vent-Lock 1+n(1) circuit and components}

1. Vent-Lock circuit components

- Manometer (Ambu Disposable Pressure Manometer, Ambu, Copenhagen, Denmark)

- One-way valves (22F x 22M, REF 50245, Mallinckrodt Pharmaceuticals)

- Disposable bacteria filters (BSF104, Vincent Medical)

- Ventilator tubing (SKU: 999027588, Hudson Rci).

2. Ventilator (Puritan Bennett 840 Ventilator System, Avante Health Solutions) on pressure control mode of ventilation (Volume Ventilation Plus ${ }^{\text {TM }}$, Avante Health Solutions) 
3. Test lungs (Standard patient: $\mathrm{R}_{\mathrm{p}}=2 \mathrm{cmH}_{2} \mathrm{O} / \mathrm{L} / \mathrm{s}$, RespiTrainer Advance, QuickLung, IngMar Medical; Variable patient: $\mathrm{R}_{\mathrm{p}}=50 \mathrm{cmH}_{2} \mathrm{O} / \mathrm{L} / \mathrm{s}$, ASL 5000, IngMar Medical).

\section{In vivo swine studies.}

1. SS11LB airflow transducer (Biopac; Goleta, CA) with BSL 4.1.3 software (Biopac; Goleta, CA).

2. MATLAB script (MATLAB 2019b, The MathWorks, Inc., Natick, MA)] is available for download of all describe analysis.

\section{Procedure}

\section{D printing procedure.}

1. Download 3D print files of the Vent-Lock splitters, flow regulators, and manometer adaptors. Print files were generated by CAD drawings (SolidWorks, Dassault Systèms) and converted into G-code using the printer's accompanying software package (PreForm, Formlabs). 3D Print Files are available upon request.

2. Open 3D print files in Solidworks

3. Print via stereolithography (Form 2, Form 3, or Form 3B, Formlabs) at 50 um layer resolution, using surgical guide resin (Surgical Guide, Formlabs). Generate support structures through PreForm where needed. Orient components in such a way that crucial surfaces such as threads or O-ring ledges are not impacted by support structures.

4. Post-process the prints by washes ( 2 cycle with 15 min per cycle) in $>99.5 \%$ isopropyl alcohol (CAS Number: $67-63-0$, Sigma Aldrich), followed by air-drying at $22^{\circ} \mathrm{C}$ for 30 minutes, and post-cure for 30 minutes with heat $60^{\circ} \mathrm{C}$ for the Form 2 printer and $70^{\circ} \mathrm{C}$ for the Form 3B printer at $405 \mathrm{~nm}$ of light (Form Cure, Formlabs).

5. If printing the FloRest, insert O-rings (E1000-212/AS568-212, O-Rings EPDM, FDA EPDM, Marco Rubber \& Plastics, Seabrook, New Hampshire, USA) to improve sealing. Production via fused deposition modeling (FDM) (e3d, BigBox3D Ltd, Oxfordshire, UK; Little Monster, Tevo 3D Electronic Technology Co. Ltd, Zhanjiang, China)

6. If printing via FDM, use PETG Filament (PETG 3D Printer Filament, FilaMatrix, Virginia, USA). Set print settings to $0.2 \mathrm{~mm}$ layer height with $30 \%$ infill, nozzle temperature of $250^{\circ} \mathrm{C}$, and bed temperature of 70 ${ }^{\circ} \mathrm{C}$; generate supports from the build platform, with no interior supports. 


\section{Sterilization}

1. 3D-printed parts produced from surgical guide resin can be sterilized by dry vacuum autoclave ( $\mathrm{Sr}$ 24C Adv-Plus ${ }^{T M}$, Consolidated Sterilizer Systems, Boston, Massachusetts, USA), 3 cycles at $120.0^{\circ} \mathrm{C}, 20$ minutes sterilization time and 20 minutes dry time.

2. 3D-printed parts produced from surgical guide resin can be sterilized by soaking in $>99.5 \%$ isopropyl alcohol (CAS Number: 67-63-0, Sigma Aldrich) for 30 minutes, air-dried at $22^{\circ} \mathrm{C}$ for 30 minutes, and placed in an oven at $40^{\circ} \mathrm{C}$ in humidified air for 48 hours (V01824HPC, Lindberg/Blue M Vacuum Oven 127.4L, Thermo Scientific, Waltham, MA, USA).

3. To check particle emission pre and post-sterilization, conduct particle count using a particle counter (SOLAIR 3100, Lighthouse Worldwide Solutions), detecting sizes 0.3 to 10 microns, for 1-minute cycles.

4. To check particle emission in humidified air, place part in humidified warm air at $40{ }^{\circ} \mathrm{C}$. Repeat step above to assess particle emission.

\section{Vent-Lock $1+n(1)$ circuit and components.}

1. Acquire Vent-Lock circuit components as assembled in Fig. 1. Vent-Lock 3DP splitters, flow regulator, and manometer adaptors were used. Commercial components include manometer (Ambu Disposable Pressure Manometer, Ambu, Copenhagen, Denmark), one-way valves (22F x 22M, REF 50245, Mallinckrodt Pharmaceuticals), disposable bacteria filters (BSF104, Vincent Medical), and ventilator tubing (SKU: 999027588, Hudson Rci).

2. Adjust ventilator settings. Place the ventilator (Puritan Bennett 840 Ventilator System, Avante Health Solutions) on pressure control mode of ventilation (Volume Ventilation Plus ${ }^{\mathrm{TM}}$, Avante Health Solutions) with additional settings detailed in Fig 2.

3. Connect circuit as depicted in Fig 1. to two test lungs (Standard patient: $\mathrm{R}_{\mathrm{p}}=2 \mathrm{cmH}_{2} \mathrm{O} / \mathrm{L} / \mathrm{s}$, RespiTrainer Advance, QuickLung, IngMar Medical; Variable patient: $\mathrm{R}_{\mathrm{p}}=50 \mathrm{cmH}_{2} \mathrm{O} / \mathrm{L} / \mathrm{s}, A S L 5000$, IngMar Medical).

4. Collect intrapulmonary data for both patients: peak inspiratory pressures, tidal volumes, and peak end expiratory pressures. Collect five total values of tidal volume per data set, to be averaged. Also collect corresponding ventilator data, including total expiratory volumes, peak inspiratory pressures, mean inspiratory pressures, and peak end expiratory pressures.

\section{In vivo swine studies.}


1. Confirm that in vivo swine studies are compliant with the Guide for the Care and Use of Laboratory Animals, and approved by the Institutional Animal Care and Use Committee by your institution.

2. Acquire two domestic swine (Sus scrofa domesticus, Oak Hill Genetics, Ewing, IL), and allow minimum of seven day acclimation period.

3. Sedate swines with a telazol, ketamine, xylazine cocktail, and intubate with a 7.0 endotracheal tube. Maintain anesthesia with isoflurane.

4. Perform femoral venous and arterial catheterization. Maintain standard ASA monitoring throughout the experiment.

5. Connect the two swines to a single ventilator or anesthesia gas machine (Drager Narkomed 2A) using the Vent-Lock circuit a depicted in Figure 1.

6. Measure flow from each expiatory limb with a SS11LB airflow transducer (Biopac; Goleta, CA). Collect flow data at 2kHz using an MP36 data acquisition unit and BSL 4.1.3 software (Biopac; Goleta, $\mathrm{CA})$.

7. Smooth the spirometry data was then smoothed with a $0.25 \mathrm{sec}$ wide moving median filter after removal of instrument noise below $0.08 \mathrm{~L} / \mathrm{sec}$ (determined by histogram inspection). Numerically integrate the smoothed data to estimate respiratory tidal volume and use a first order numeric derivative to calculate the instantaneous respiratory rate. Determine the noise floor for the integrated volume by histogram inspection: the resulting threshold should approximate $90 \mathrm{~mL}$.

8. Align the anesthesia record and the spirometry results using common timestamps. Remove all breaths spontaneously initiated by the swine (identified by respiratory rates more than $30 \%$ away from the ventilator set point) from analysis.

9. Calculate the mean and standard deviation for each anesthesia record entry for respiratory rate, tidal volume, minute ventilation, and lung compliance.

10. A custom MATLAB script (MATLAB 2019b, The MathWorks, Inc., Natick, MA)] is available for download of all describe analysis.

11. Collect arterial and venous blood gas data every 15 minutes following any changes to the Vent-Lock 3DP device.

12. Following the procedure, euthanize the swine with an overdose $(\sim 150 \mathrm{mg} / \mathrm{kg})$ of supersaturated potassium chloride IV while under anesthesia.

13. Perform necropsy to assess for any gross lung pathology.

\section{Troubleshooting}




\section{Time Taken}

\section{Anticipated Results}

\section{References}

1. Poston, J. T., Patel, B. K. \& Davis, A. M. Management of Critically III Adults With COVID-19. JAMA 323, 1839-1841 (2020).

2. GlobalData. 880,000 global ventilator shortage can only be met by new simple design requiring minimal operator training time, says GlobalData. https://www.globaldata.com/880000-global-ventilatorshortage-can-only-be-met-by-new-simple-design-requiring-minimal-operator-training-time-says-globaldata/ (2020).

3. Oshitani, H., Kamigaki, T. \& Suzuki, A. Major Issues and Challenges of Influenza Pandemic Preparedness in Developing Countries. Emerg. Infect. Dis. J. 14, 875 (2008).

4. Murray, C. J., Lopez, A. D., Chin, B., Feehan, D. \& Hill, K. H. Estimation of potential global pandemic influenza mortality on the basis of vital registry data from the 1918-20 pandemic: a quantitative analysis. Lancet 368, 2211-2218 (2006).

5. Maclean, R. \& Marks, S. 10 African Countries Have No Ventilators. That is only part of the problem. The New York Times https://www.nytimes.com/2020/04/18/world/africa/africa-coronavirusventilators.html (2020).

6. Bartsch, S. M. et al. The Potential Health Care Costs And Resource Use Associated With COVID-19 In The United States. Health Aff. 10.1377/hlthaff (2020) doi:10.1377/hlthaff.2020.00426.

7. Ranney, M. L., Griffeth, V. \& Jha, A. K. Critical supply shortages - The need for ventilators and personal protective equipment during the Covid-19 pandemic. New England Journal of Medicine (2020) doi:10.1056/NEJMp2006141.

8. Xu, S. \& Li, Y. Beware of the second wave of COVID-19. Lancet 395, 1321-1322 (2020).

9. SCCM Rapid Cycle 3 Response Summary. Society of Critical Care Medicine (2020).

10. Sebastian, D. GM to Make 30,000 Ventilators for National Stockpile in \$489 Million Contract. Wall Street Journa/ https://www.wsj.com/articles/gm-to-make-30-000-ventilators-for-national-stockpile-in-489million-contract-11586347203 (2020).

11. United States Resource Availability for COVID-19. The Society of Critical Care Medicine https://sccm.org/Blog/March-2020/United-States-Resource-Availability-for-COVID-19 (2020). 
12. Mirchandani, P. Health Care Supply Chains: COVID-19 Challenges and Pressing Actions. Ann. Intern. Med. (2020).

13. Neyman, G. \& Irvin, C. B. A Single Ventilator for Multiple Simulated Patients to Meet Disaster Surge. Acad. Emerg. Med. 13, 1246-1249 (2006).

14. Menes, K., Tintinalli, J. \& Plaster, L. How One Las Vegas ED Saved Hundreds of Lives After the Worst Mass Shooting in U.S. History. Emergency Physicians Monthly https://epmonthly.com/article/notheroes-wear-capes-one-las-vegas-ed-saved-hundreds-lives-worst-mass-shooting-u-s-history/ (2017).

15. Chatburn, R. L., Branson, R. D. \& Hatipoğlu, U. Multiplex Ventilation: A Simulation-based Study of Ventilating Two Patients with One Ventilator. Respir. Care respcare.07882 (2020) doi:10.4187/respcare.07882.

16. Consensus Statement on Multiple Patients Per Ventilator. The Society of Critical Care Medicine https://www.sccm.org/Disaster/Joint-Statement-on-Multiple-Patients-Per-Ventilato (2020).

17. Srinivasan, S. et al. Individualized System for Augmenting Ventilator Efficacy (iSAVE): A Rapidly deployable system to expand ventilator capacity. bioRxiv 2020.03.28.012617 (2020) doi:10.1101/2020.03.28.012617.

18. Impact of the Global Medical Supply Chain on SNS Operations and Communications. Impact of the Global Medical Supply Chain on SNS Operations and Communications (2018). doi:10.17226/25149.

19. Boyson, S. A defining moment in our medical supply chain crisis. The Hill https://thehill.com/opinion/international/490963-a-defining-moment-in-our-medical-supply-chain-crisis (2020).

20. Attaran, M. \& Attaran, M. 3D Printing Role in Filling the Critical Gap in the Medical Supply Chain during COVID-19 Pandemic. Am. J. Ind. Bus. Manag. 10, 988-1001 (2020).

21. Zadpoor, A. A. Design for additive bio-manufacturing: From patient-specific medical devices to rationally designed meta-biomaterials. International Journal of Molecular Sciences (2017) doi:10.3390/ijms18081607.

22. FDA. Technical Considerations for Additive Manufactured Devices Draft Guidance for Industry and Food and Drug Administration Staff. Clim. Chang. 2013 - Phys. Sci. Basis (2016) doi:10.1017/CB09781107415324.004.

23. Ishack, S. \& Lipner, S. R. Applications of 3D Printing Technology to Address COVID-19 Related Supply Shortages. Am. J. Med. (2020) doi:10.1016/j.amjmed.2020.04.002.

24. APSF/ASA. APSF/ASA Guidance on Purposing Anesthesia Machines as ICU Ventilators. Am. Soc. Anesthesiol. (2020). 
25. Rosenthal, B. M., Pinkowski, J. \& Goldstein, J. 'The Other Option Is Death': New York Starts Sharing of Ventilators. The New York Times https://www.nytimes.com/2020/03/26/health/coronavirus-ventilatorsharing.html (2020).

26. Ibrahim, A. M. S. et al. Three-dimensional Printing in Developing Countries. Plast. Reconstr. Surg. Glob. Open 3, (2015).

\section{Acknowledgements}

ACKNOWLEDGEMENTS We would like to thank the Johns Hopkins Medicine Simulation Center Jordan Duval-Arnould, Julie Perretta, and Joe Dwyer for use of the simulation center; Dr. Thao (Vicky) Nguyen, Dr. Joe Katz for their advice; Dr. Adam Sapirstein for their medical expertise; Dr. Sarah Clever for her support; the Johns Hopkins Applied Physics Lab for guidance and advice; Dr. Gerald Brandacher and Yi-nan Guo for use of their autoclave; Gregory Bova for loan of his particle counter; and Dr. Arun Agrawal and Azra Horowitz for their advice on ventilator splitting. The authors acknowledge financial support from the Johns Hopkins University President's Response to COVID-19 Fund, the Start-Up Fund from the Whiting School of Engineering at Johns Hopkins University, support from the Department of Civil and Systems Engineering and Johns Hopkins Center for Additive Manufacturing and Architected Materials, and the National Science Foundation (DMREF-1628974). JU acknowledges financial support from the U.S. Army Research Office (ARO) sponsored NDSEG Fellowship program and AT acknowledges support by a NASA Space Technology Research Fellowship.

\section{Figures}




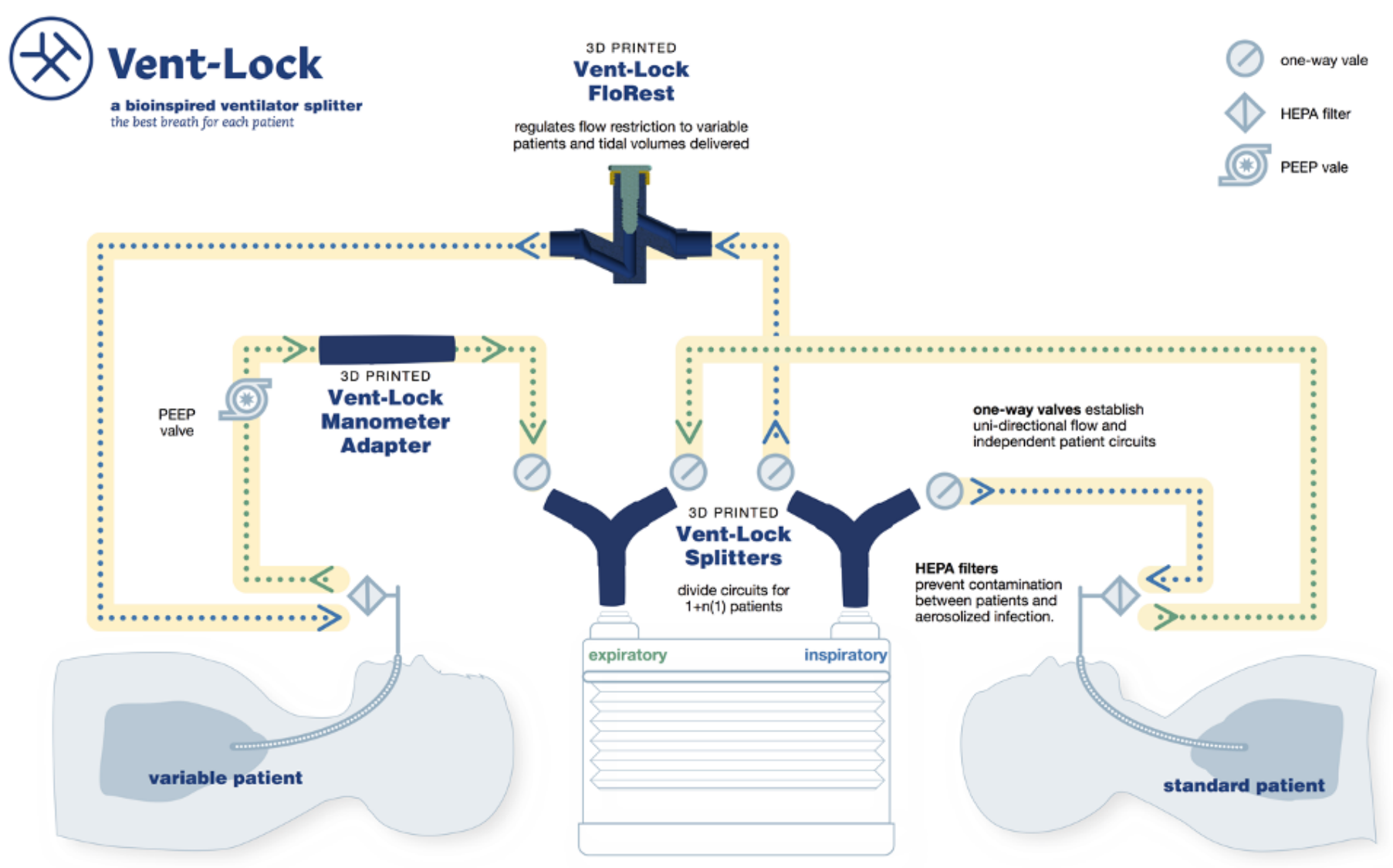

\section{Figure 1}

Vent-Lock ventilator multiplexing 1+n(1) circuit and 3DP components. Our 1+n(1) circuit proposes having a standard patient with minimal features, thus are ventilated per ventilator settings. Additional patients added to the circuit will be considered $\mathrm{n}(1)$, and will have variable flow and PEEP as controlled by circuit components. Please note that all patients, regardless of standard or variable, have one-way (check) valves and filters. 


\begin{tabular}{|l|l|}
\hline \multicolumn{2}{|l}{ Ventilator and Ventilator Alarm Settings } \\
\hline ventilator parameter & value \\
\hline pressure (on pressure control mode) & $25 \mathrm{cmH} 2 \mathrm{O}$ \\
\hline volume (on volume control mode) & $2 \mathrm{~L}$ for 2 patients \\
\hline PEEP & $5 \mathrm{cmH} 2 \mathrm{O}$ \\
\hline respiratory rate & 8 \\
\hline I/E ratio & 0.8 \\
\hline ventilator alarm settings & \\
\hline tidal volume & value \\
\hline pressures & $150 \mathrm{~mL}$ \\
\hline breath trigger & $60 \mathrm{to} 70 \mathrm{cmH} 2 \mathrm{O}$ \\
\hline
\end{tabular}

\section{Figure 2}

Ventilator and ventilator alarm settings on 840 Ventilator System, Nellcor Puritan Bennett. Ventilator settings used in testing the Vent-Lock ventilator multiplexing system in the simulation center and with swine studies. Alarm trigger settings on the ventilator; please note that alarm triggers are set to be at far extremes to prevent ventilator from terminating ventilation, or from one patient triggering a breath for the entire circuit.

\section{Supplementary Files}

This is a list of supplementary files associated with this preprint. Click to download.

- splitvent.m 